\title{
Spermidine promotes retinal ganglion cell survival and optic nerve regeneration in adult mice following optic nerve injury
}

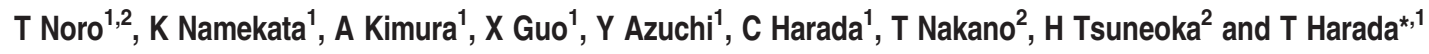

Spermidine acts as an endogenous free radical scavenger and inhibits the action of reactive oxygen species. In this study, we examined the effects of spermidine on retinal ganglion cell (RGC) death in a mouse model of optic nerve injury (ONI). Daily ingestion of spermidine reduced RGC death following ONI and sequential in vivo retinal imaging revealed that spermidine effectively prevented retinal degeneration. Apoptosis signal-regulating kinase-1 (ASK1) is an evolutionarily conserved mitogenactivated protein kinase kinase kinase and has an important role in ONI-induced RGC apoptosis. We demonstrated that spermidine suppresses ONI-induced activation of the ASK1-p38 mitogen-activated protein kinase pathway. Moreover, production of chemokines important for microglia recruitment was decreased with spermidine treatment and, consequently, accumulation of retinal microglia is reduced. In addition, the ONI-induced expression of inducible nitric oxide synthase in the retina was inhibited with spermidine treatment, particularly in microglia. Furthermore, daily spermidine intake enhanced optic nerve regeneration in vivo. Our findings indicate that spermidine stimulates neuroprotection as well as neuroregeneration, and may be useful for treatment of various neurodegenerative diseases including glaucoma.

Cell Death and Disease (2015) 6, e1720; doi:10.1038/cddis.2015.93; published online 16 April 2015

Traumatic optic neuropathy is a common clinical problem that occurs in $0.5-5 \%$ of patients with closed head injury. ${ }^{1}$ A damage to the optic nerve causes shear stress and induces secondary swelling within the optic canal, accompanied by subsequent RGC loss and optic nerve atrophy. ${ }^{2}$ Although no large natural history or randomized controlled trial has been published, neither corticosteroid therapy nor optic canal decompression surgery is considered as standard treatments for patients with traumatic optic neuropathy, ${ }^{3}$ and there is a lack of effective treatment at present. Research into finding therapeutic targets for treatment of traumatic optic neuropathy indicated that neuroprotection and axon regeneration may be effective strategies and studies using an optic nerve injury (ONI) model in rodents have provided useful information. For example, neurotrophins, such as brain-derived neurotrophic factor and ciliary neurotrophic factor, protect retinal ganglion cells (RGCs) and promote axon regeneration in an ONI model. ${ }^{4-6}$ In addition, inhibition of neuroinflammatory events such as upregulation of tumor necrosis factor (TNF)- $a$ and nitric oxide synthase (NOS) may be effective for RGC protection following $\mathrm{ONI}^{7}$ The ONI model mimics some aspects of glaucoma, including RGC death induced by excitotoxicity and oxidative stress, and therefore it is also a useful animal model for glaucoma.

Glaucoma is one of the leading causes of vision loss in the world and it is estimated that this condition will affect more than
80 million individuals worldwide by 2020 , with at least $6-8$ million individuals becoming bilaterally blind. ${ }^{8}$ Glaucoma is characterized by progressive degeneration of RGCs and their axons, which are usually associated with elevated intraocular pressure, but there is a subset of glaucoma termed normal tension glaucoma (NTG) that presents with statistically normal intraocular pressure. There are several animal models of glaucoma, including DBA/2J mice, ${ }^{9}$ and inducible models such as cauterization of episcleral veins. ${ }^{10-12}$ In addition, we previously reported that loss of glutamate transporters (EAAC1 or GLAST) in mice leads to RGC degeneration that is similar to NTG ${ }^{13}$ and these animal models have been useful in examining potential therapeutic targets. ${ }^{14-16}$

Spermidine is naturally and almost exclusively accumulated in glial cells in the brain and retina. ${ }^{17,18}$ It acts as an endogenous free radical scavenger and inhibits the action of reactive oxygen species. Indeed, it has been reported that spermidine has key roles in mediating protection against oxidative damage caused by hydrogen peroxide in cultured mouse fibroblasts ${ }^{19}$ and administration of spermidine extended the lifespan of yeast, flies, worms and human immune cells by upregulating the lysosomal/vacuolar degradation pathway, referred to as autophagy, which leads to enhanced resistance to oxidative stress and decreased cell death. ${ }^{20}$ Previously, we reported that oral administration of spermidine ameliorates severity of experimental autoimmune

\footnotetext{
${ }^{1}$ Visual Research Project, Tokyo Metropolitan Institute of Medical Science, Tokyo, Japan and ${ }^{2}$ Department of Ophthalmology, The Jikei University School of Medicine, Tokyo, Japan

*Corresponding author: T Harada, Visual Research Project, Tokyo Metropolitan Institute of Medical Science, 2-1-6 Kamikitazawa, Setagaya-ku, Tokyo 156-8506, Japan. Tel: +81 36834 2338; Fax: +81 36834 2339; E-mail: harada-tk@igakuken.or.jp

Abbreviations: ASK1, apoptosis signal-regulating kinase 1; CTB, cholera toxin $\beta$-subunit; GCC, ganglion cell complex; GCL, ganglion cell layer; IP, immunopositive; INL, inner nuclear layer; iNOS, inducible nitric oxide synthase; IRL, inner retinal layer; MCP-1, monocyte chemoattractant protein-1; MIP-1 $\alpha$, macrophage inflammatory protein $1 \alpha$; NOS, nitric oxide synthase; NTG, normal tension glaucoma; OCT, optical coherence tomography; ONI, optic nerve injury; ONL, outer nuclear layer; RANTES, regulated on activation normal T-cell expressed and secreted; RGC, retinal ganglion cell; TNF- $\alpha$, tumor necrosis factor- $\alpha$

Received 12.12.14; revised 13.2.15; accepted 02.3.15; Edited by A Verkhratsky
} 
encephalomyelitis, an animal model of multiple sclerosis, by suppression of oxidative stress, ${ }^{21}$ suggesting that spermidine may also be effective in protecting RGCs from increased oxidative stress associated with various pathogenic conditions in the eye including traumatic optic neuropathy and glaucoma.

In this study, we examined the effects of daily spermidine intake on ONI-induced retinal degeneration. We monitored changes in retinal morphology over a course of 2 weeks following ONI, using optical coherence tomography (OCT), which permits noninvasive, longitudinal and quantitative assessment of retinal structures in living animals. We also explored possible mechanisms associated with spermidinemediated neuroprotection.

\section{Results}

Spermidine prevents RGC death following ONI. We first investigated neuroprotective effects of spermidine in the retina following ONI. Spermidine $(30 \mathrm{mM})$ was given to adult mice in their drinking water for 18 days starting from 4 days before ONI through to 14 days after ONI (Figure 1a). By day 7 (d7) and d14 following ONI, the number of RGCs was significantly reduced, but spermidine treatment suppressed such RGC loss (Figures $1 \mathrm{~b}$ and $\mathrm{c}$ ). In addition, the thickness of the inner retinal layer (IRL) was significantly decreased in vehicle-treated mice following ONI, whereas this effect was prevented in spermidine-treated mice (Figure 1d). These results suggest that spermidine protects RGCs from ONIinduced cell death.

\section{Spermidine suppresses retinal degeneration following} ONI. We also visualized retinal layers using OCT, a noninvasive imaging technique that can be used to acquire crosssectional tomographic images of the retina in vivo. ${ }^{22}$ This technique is very useful in monitoring the changes in retinal structures over a period of time following injury in living animals. ${ }^{15,16,22-24}$ The average thickness of the ganglion cell complex (GCC), which includes the nerve fiber layer, ganglion cell layer (GCL) and inner plexiform layer, was decreased in vehicle-treated mice over the course of 2 weeks following $\mathrm{ONI}$, and this effect was milder in spermidine-treated mice (Figure 2a). For quantitative analyses, GCC was measured by scanning the retina in a circle, centering around the optic nerve disc (Figure $2 b$ ), and the average GCC thickness was determined from acquired images (Figure 2c). The quantitative analyses confirmed that the reduction in the GCC thickness following $\mathrm{ONI}$ is significantly suppressed with spermidine treatment (Figure 2d). These results suggest that spermidine suppresses ONI-induced retinal degeneration.

\section{Spermidine inhibits activation of the ASK1-p38 pathway in RGCs following ONI. We recently reported that the apoptosis signal-regulating kinase-1 (ASK1)-p38 pathway is involved in ONI-induced RGC death. ${ }^{22}$ ASK1 has key roles in human diseases closely related to dysfunction of cellular responses to oxidative stress and endoplasmic reticulum stressors, including neurodegenerative diseases. ${ }^{14,25}$ As spermidine possesses anti-oxidant properties, we examined whether spermidine modulates the ASK1-p38 pathway.}

Following ONI, the retinal expression of phosphorylated (phospho)-ASK1 and p38 were significantly increased, but spermidine inhibited these changes (Figures $3 a$ and b). Immunohistochemical analyses revealed that the increase in phospho-p38 expression following ONI was detected mainly in the GCL, which was doubled-labeled with NeuN (a RGC marker) (Figure 3c). Spermidine treatment clearly suppressed this increased expression levels of phosphop38 (Figures $3 d$ and e). These results suggest that spermidine inhibits ONI-induced activation of the ASK1-p38 pathway in RGCs, which leads to RGC protection.

Spermidine decreases chemokine productions and microglial accumulation following ONI. We explored other potential mechanisms associated with spermidine-mediated neuroprotection. We previously reported that $\mathrm{ONI}$ increases retinal expressions of various chemokines implicated in the pathogenesis of neuroinflammation. ${ }^{22}$ Therefore, we examined the ONI-induced changes in monocyte chemoattractant protein-1 (MCP-1), macrophage inflammatory protein $1 a$ $(\mathrm{MIP}-1 a)$ and regulated on activation, normal T-cellexpressed and -secreted (RANTES) expression levels in the retina. As expected, the mRNA levels of MCP-1, MIP-1a and RANTES were all increased in the retina following ONI. We found that the expressions of examined chemokines were also elevated in the spermidine-treated mouse retina, but the extent of increase was significantly less for MCP-1 and RANTES (Figure 4a). Spermidine did not affect the retinal MIP-1a expression level. As MCP-1 and RANTES are involved in migration and activation of microglia, we examined the presence of microglia in the retina following $\mathrm{ONI}$ with or without spermidine treatment. Immunohistochemical analyses revealed that $\mathrm{ONI}$ induced a significant increase in the number of microglia in the retina at $\mathrm{d} 7$ and spermidine treatment almost completely inhibited this effect (Figures $4 b$ and $c$ ). These results suggest that spermidine suppresses production of chemokines important for activation and migration of microglia, resulting in RGC protection in the ONI model.

Spermidine suppresses the inducible NOS expression in microglia following ONI. We next investigated whether spermidine affects any cellular events within microglia. As the inducible NOS (iNOS) expression level is upregulated in activated microglia, which can cause RGC death, ${ }^{26,27}$ we examined whether spermidine modulates the level of microglial iNOS expression following ONI. We first found that ONI induced a significant increase in the iNOS expression level in the whole retina, which was suppressed with spermidine treatment (Figure 5a). Immunohistochemical analyses revealed that ONI increased the number of immunopositive (IP) cells for both iba1 and iNOS in the inner retina and the extent of increase was less in the spermidine-treated retina (Figure 5b). The iNOS-IP cells were double labeled with iba1, suggesting that iNOS is produced in microglia in response to ONI, which is detrimental to RGC survival, and spermidine exerts neuroprotective effects by reducing the number of activated microglia and suppressing iNOS upregulation. 


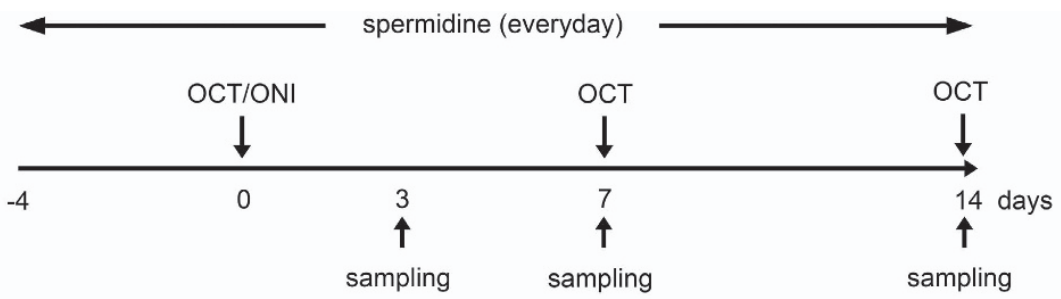

b
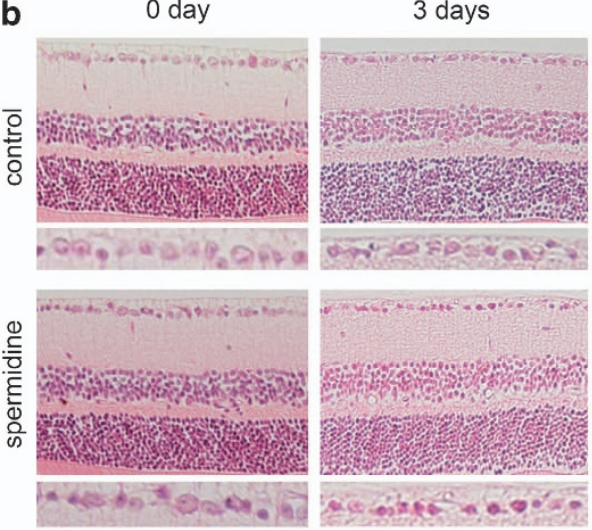

C

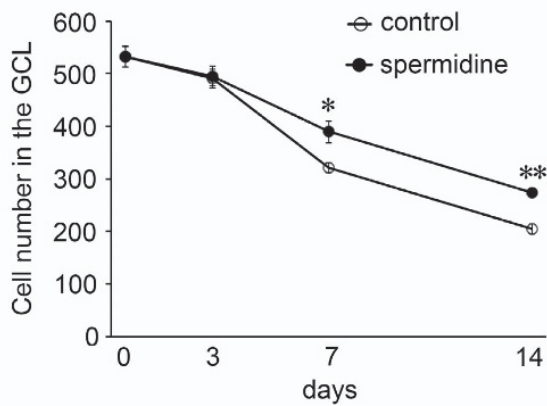

7 days

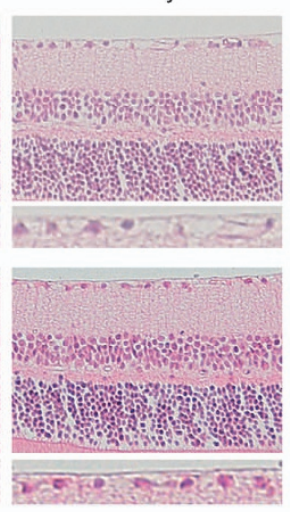

14 days

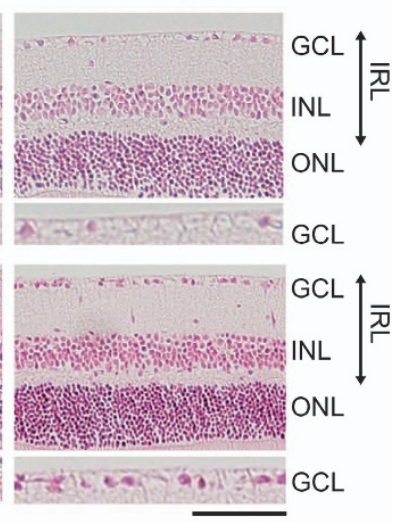

d

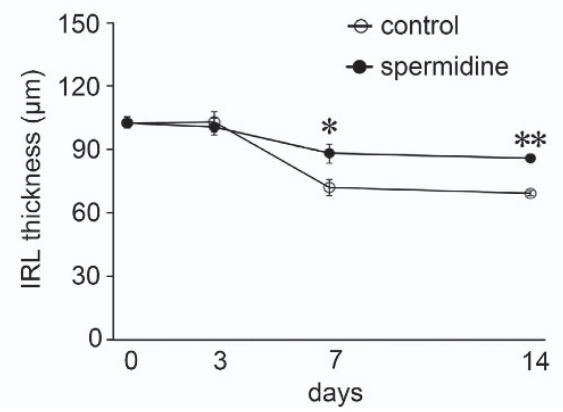

Figure 1 Effects of spermidine on RGC loss and IRL thickness following ONI. (a) Experimental protocols. Spermidine ( $30 \mathrm{mM}$ ) was added to drinking water for the treatment group from 4 days before ONI throughout the experimental period and the control group received normal drinking water. The treatment period was completed on d14 after ONI. (b) Retinal sections stained with hematoxylin and eosin at d3,7 and 14 after ONI in control and spermidine-treated mice. Scale bar: 100 and $50 \mu \mathrm{m}$ in the upper and immediately lower panels, respectively. GCL, ganglion cell layer; INL, inner nuclear layer; IRL, inner retinal layer; ONL, outer nuclear layer. Quantification of the RGC number (c) and IRL thickness (d) in control and spermidine-treated mice. The data are presented as means \pm S.E.M. of six samples for each experiment. ${ }^{*} P<0.05,{ }^{* *} P<0.01$

Spermidine enhances optic nerve regeneration. As spermidine promotes RGC survival following ONI, we hypothesized that it may have beneficial effects on optic nerve regeneration. Optic nerve regeneration is an important area of research for development of treatment strategies for conditions that damage the optic nerve, as well as the retina, including glaucoma. The regenerating axons of RGCs were traced by injecting cholera toxin $\beta$-subunit (CTB) conjugated to Alexa 488 into the vitreous of the retina at d11 after ONI (Figure 6a). Following $\mathrm{ONI}$ at $\mathrm{d} 14$, the number of regenerating axons in spermidine-treated mice was much greater than the control mice (Figures $6 a$ and b). The quantitative analyses demonstrated that the number of regenerating axons that extended beyond $250 \mu \mathrm{m}$ was approximately 45 in control compared with about 120 with spermidine treatment, and half of the axons in each conditions extended beyond $500 \mu \mathrm{m}$. These results suggest that spermidine enhances the number of regenerating axons, probably due to the increased RGC survival rate, but it may have a limited effect on the regenerative ability of axons.

\section{Discussion}

In this study, we reported that oral administration of spermidine exerts neuroprotective effects in an ONI model. We demonstrated that daily ingestion of spermidine protects RGCs from $\mathrm{ONI}$-induced cell death and retinal degeneration. We also showed that spermidine suppresses ONI-induced activation of the ASK1-p38 pathway in RGCs, which is one of the potential mechanisms involved in spermidine-mediated neuroprotection. In addition, we showed that production of chemokines important for recruitment of microglia is decreased with spermidine treatment and, consequently, accumulation of retinal microglia is reduced. ONI-induced iNOS expression in the retina was inhibited with spermidine treatment, in particular in microglia. Furthermore, daily spermidine intake enhanced 
a 0 day 7 days 14 days
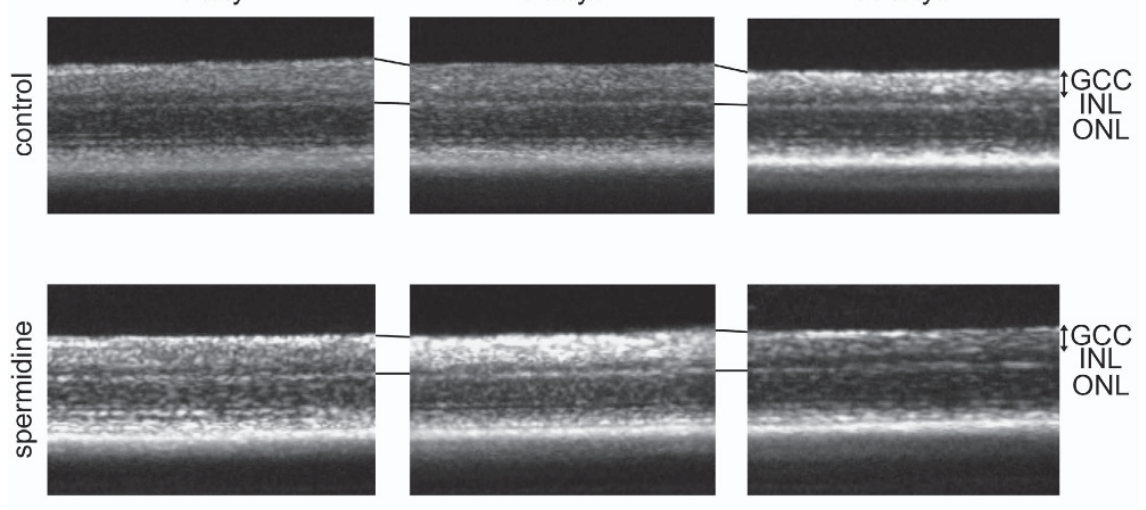

b

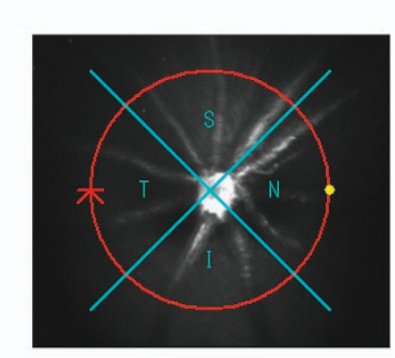

d

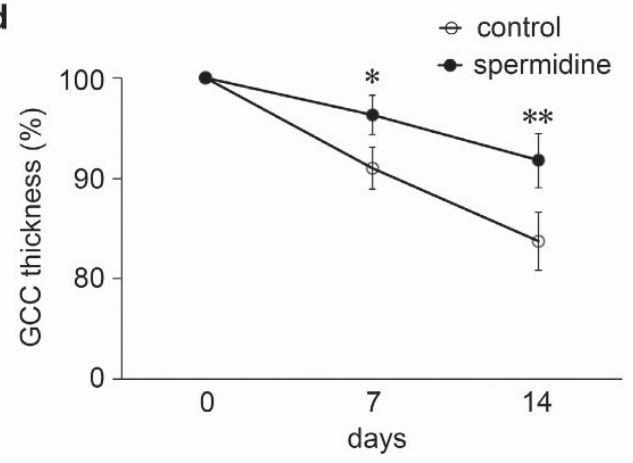

c

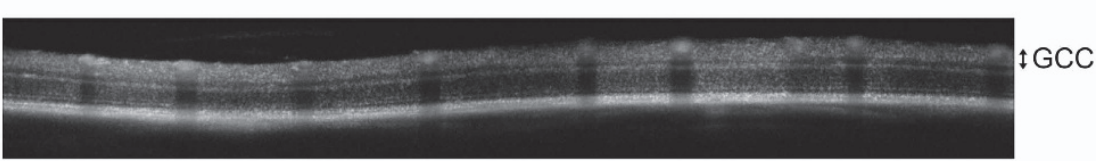

Figure 2 In vivo imaging of the retina in control and spermidine-treated mice. (a) OCT cross-sectional images of retinas at d0, 7 and 14 following ONI. GCC, ganglion cell complex. (b) An image of a circle centering around the optic nerve disc. (c) An OCT circular scan image captured from b. (d) Longitudinal evaluation of the GCC thickness by a circular scan. The data are presented as means \pm S.E.M. of six samples for each experiment. ${ }^{*} P<0.05,{ }^{* *} P<0.01$

optic nerve regeneration in vivo, suggesting that spermidine may stimulate neuroprotection as well as neuroregeneration, and may be useful for treatment of various conditions including traumatic optic neuropathy and glaucoma.

The ASK1-p38 pathway is activated in response to multiple types of stress, such as oxidative stress and endoplasmic reticulum stress. ${ }^{28}$ It is implicated in diseases including cancer, Alzheimer's disease and multiple sclerosis. ${ }^{29}$ It also has a role in RGC death and optic nerve degeneration induced under various conditions. ${ }^{14,22,30,31}$ Considering the antioxidant properties of spermidine, it is plausible that spermidine suppressed ONI-induced activation of the ASK1-p38 pathway in RGCs, because it reduces the oxidative stress level. ${ }^{19-21}$ Oxidative stress is an important risk factor in human glaucoma $^{32}$ and an antioxidant, $a$-lipoic acid, delivered through diet protects RGCs in DBA/2 J mice, an animal model that recapitulates the slow, progressive nature of human glaucoma. ${ }^{33}$ It would be interesting to investigate whether spermidine intake is also beneficial in animal models of glaucoma, including NTG. ${ }^{13}$

Microglia are resident macrophages in the central nervous system and retina, and are activated after injury. Activated microglia produce cytotoxic substances, such as TNF- $a$, reactive oxidative species, nitric oxide, proteases and excitatory amino acids that may induce neuronal cell death. These processes are implicated in various neurodegenerative diseases including glaucoma and Alzheimer's disease. ${ }^{34,35} \mathrm{We}$ previously demonstrated that MCP-1 is upregulated in RGCs following $\mathrm{ONI},{ }^{22}$ and in this study we showed that spermidine reduces ONI-induced upregulation of MCP-1 and RANTES, another chemotactic molecule for microglia, resulting in inhibition of microglia accumulation. As these chemokines recruit and activate microglia that result in neuronal apoptosis and exacerbation of neurodegeneration, ${ }^{36,37}$ we suggest that spermidine exerts neuroprotective effects via suppression of microglia recruitment and possibly activation. However, we cannot exclude the possibility that microglial invasion to the inner retina is also associated with molecules that are released when RGCs die, rather than just MCP-1 and RANTES, being released by living RGCs. Although we cannot discard the possibility that the role of spermidine under these conditions is to reduce the number of migrating microglia and so it may not have a direct effect on cellular events within microglia, we suggest that suppression of iNOS upregulation is one of the mechanisms for spermidine-mediated neuroprotection. Taking together, it is speculated that endogenous 
spermidine accumulated in retinal glia may be an initial resource for RGC survival, and if such resources are depleted due to aging and trauma then exogenous spermidine may protect RGCs. ${ }^{38}$ Spermidine is known to act on the neural
$\mathrm{N}$-methyl-D-aspartate receptor in a bi-phasic concentrationdependent manner: at submillimolar doses it blocks the receptors, but at submicromolar levels it activates the receptors. $^{39}$ In the current study, the concentration of a

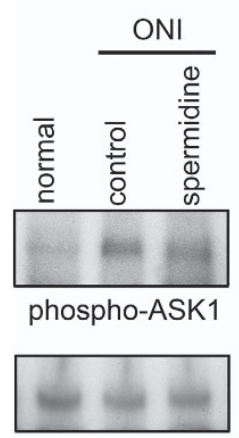

total ASK1

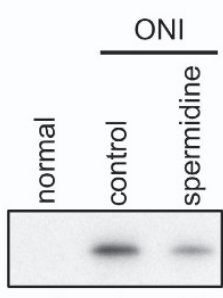

phospho-p38

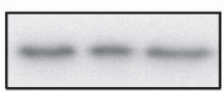

total p38

b

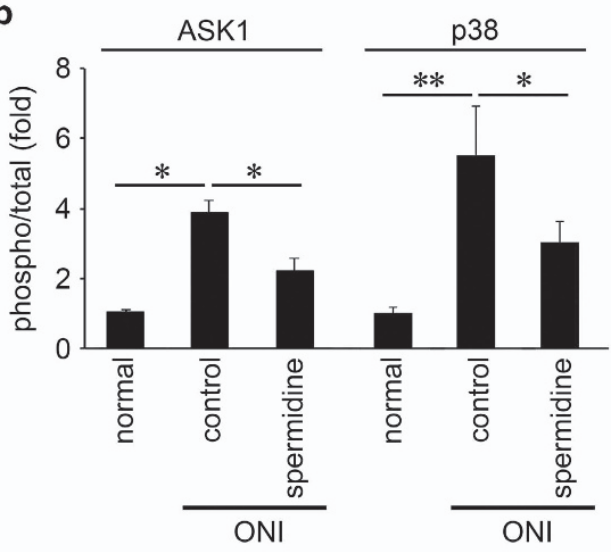

C
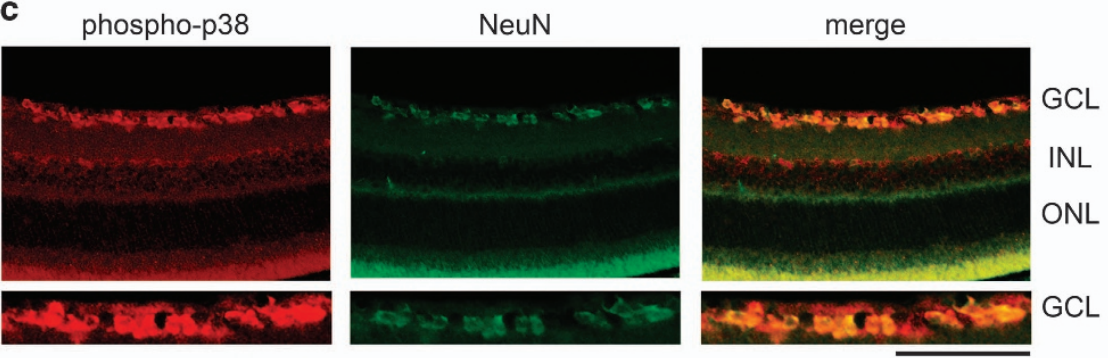

d

ONI
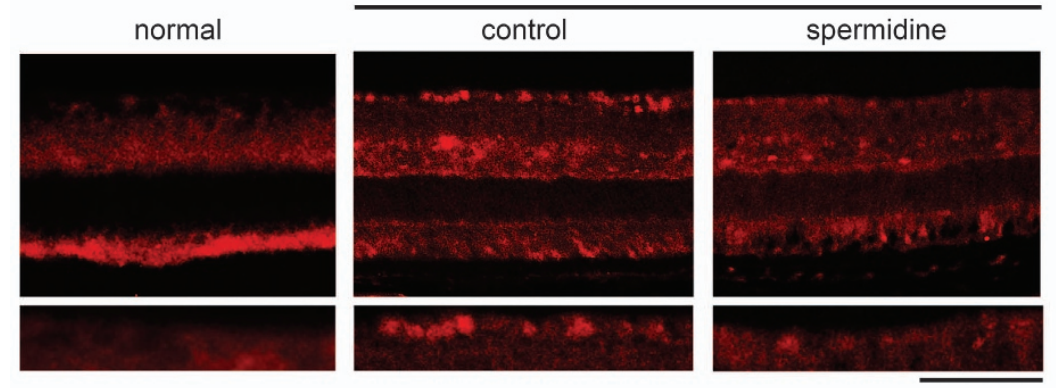

GCL

INL

ONL

GCL

e

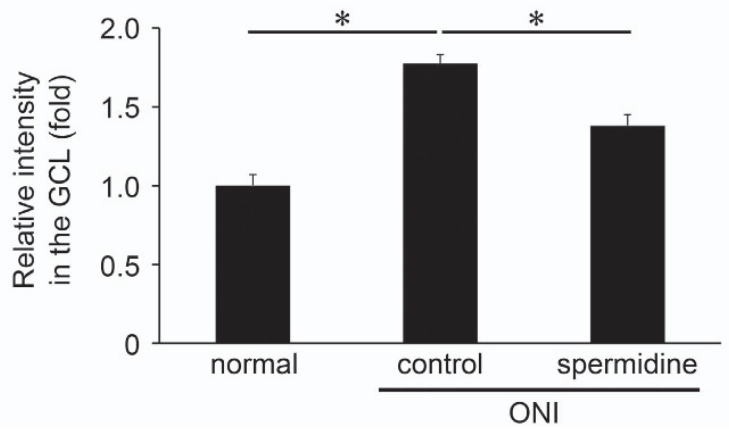

Figure 3 Effects of spermidine on ONI-induced activation of the ASK1 signaling pathway in the retina. (a) Effects of spermidine on activation of the ASK1-p38 pathway in whole retinas at $4 \mathrm{~h}$ after ONI. (b) Quantitative analyses of ONI-induced activation of ASK1 and p38 in whole retinas in a. (c) Immunohistochemical analyses of ONI-induced p38 phosphorylation (red) and NeuN expression (green) in the retina. Overlapping immunoreactivities (yellow) indicate p38 phosphorylation in RGCs. Scale bar: 200 and $100 \mu$ m in the upper and immediately lower panels, respectively. (d) Immunohistochemical analyses of ONI-induced retinal p38 phosphorylation with or without spermidine treatment. Scale bar: 200 and $100 \mu \mathrm{m}$ in the upper and immediately lower panels, respectively. (e) Quantitative analyses of $\mathbf{d}$. Data are normalized to the phospho-p38 intensity at the GCL in normal mice. The data are presented as means \pm S.E.M. of six samples for each experiment. ${ }^{*} P<0.05,{ }^{* *} P<0.01$ 
a

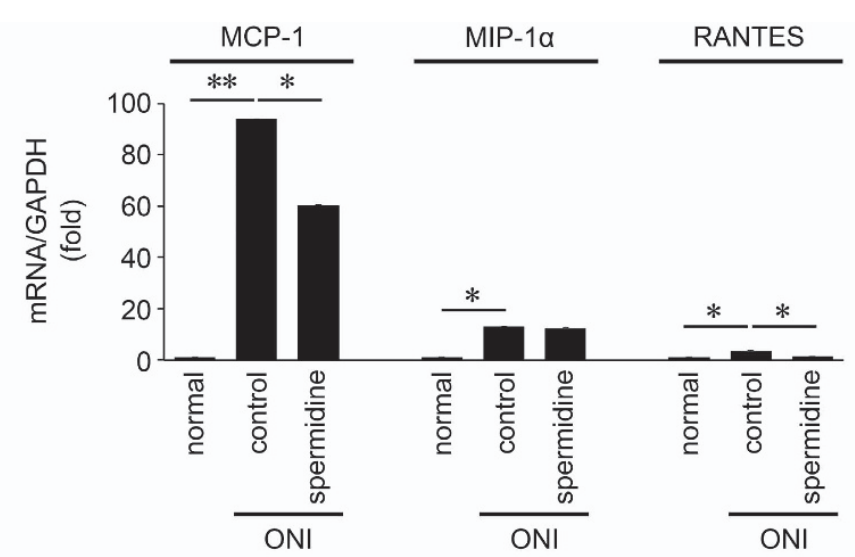

\section{b}
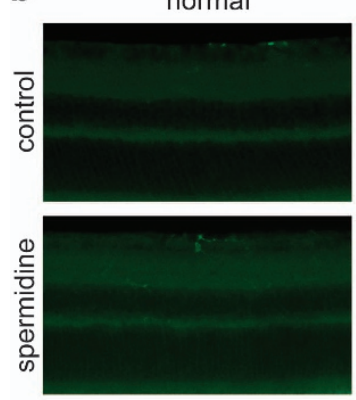

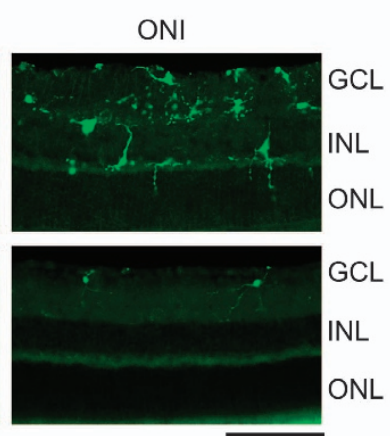

C
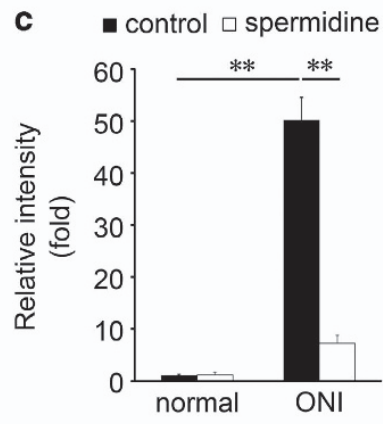

Figure 4 Effects of spermidine on ONI-induced upregulation of chemokines and accumulation of microglia. (a) mRNA expression levels of MCP-1, MIP-1 $\alpha$ and RANTES in whole retinas at $\mathrm{d} 4$ after ONI were determined using quantitative real-time PCR. Glyceraldehyde 3-phosphate dehydrogenase (GAPDH) was used as an internal control. (b) Immunohistochemical analyses of ONI-induced migration of microglia detected with an iba1 antibody in the retina at $0 \mathrm{~h}$ and d7 after ONI. Scale bar: $100 \mu \mathrm{m}$. (c) Quantitative analyses of $\mathbf{b}$. Data are normalized to the iba1 intensity in normal mice. The data are presented as means \pm S.E.M. of six samples for each experiment. ${ }^{*} P<0.05$, ${ }^{* *} P<0.01$

spermidine used was $30 \mathrm{mM}$, which is the same dose that protected RGCs in a mouse model of multiple sclerosis. ${ }^{21}$ Further studies will be required to measure the concentration of extracellular spermidine near RGCs, to ensure the best outcome from spermidine treatment.

It has been shown that glial cells can not synthesize spermidine, but exogenous spermidine from blood vessels can be transported to glia by organic cation transporters and vesicular polyamine transporters. ${ }^{40-42}$ Therefore, spermidine supplement and the effect of polyamine treatment may depend on the status of the glial cell transport systems. Furthermore, spermidine has an important role in neural cell survival and resistance against trauma by openning the connexine gap junctions between astrocytes and making the glial syncytium functional to propagate vital molecules and ions from one glial cell to another. ${ }^{43,44}$ As glia-glia and glia-neuron interactions have important roles in neuroprotection, it would be interesting to investigate whether spermidine mediates its neuroprotective effects also through stimulation or inhibition of various molecules associated with cell survival or cell death within microglia and Müller glia. ${ }^{45-47}$

Stimulation of optic nerve regeneration is an important area of research for restoring visual function after optic nerve damage. To date, studies have identified some key molecules that can enhance optic nerve regeneration and, strikingly, partial recovery of visual function following ONI has been achieved. ${ }^{48-50}$ We show that neuroregeneration is enhanced with daily spermidine intake in vivo, which is consistent with previous findings demonstrating spermidine-mediated stimulation of neurite outgrowth in vitro and in vivo. ${ }^{51}$ The significance of our current findings is that we administered spermidine by oral ingestion ad libitum, which is therapeutically more viable than by intravitreal injection that was used in the previous study. It is important to note that spermidine increased the number of regenerating axons, but the increase in length was limited. These results suggest that the effects of spermidine on axon regeneration depend on increasing the RGC survival rate, rather than enhancing the ability of axons to grow.

Spermidine is a natural component of our diet and several foods are known to contain high levels of spermidine, including soy beans, tea leaves and mushrooms. Evidence shows that eating food that is rich in spermidine results in increased blood spermidine levels, ${ }^{52}$ suggesting that beneficial effects of spermidine can be easily attained by making a conscious choice of food. A recent study has shown that putrescine, a precursor of spermidine, can be converted into $\gamma$-aminobutyric acid that makes the inhibitory pathway stronger and protects neurons from death, ${ }^{53}$ establishing a previously unknown neuroprotective role of polyamines in the developing brain. In conclusion, our findings indicate that spermidine intake exerts neuroprotective and neuroregenerative effects in the retina following injury, suggesting that polyamines including spermidine may be good therapeutic candidates for retinal and optic nerve degeneration, such as traumatic optic neuropathy and glaucoma. 
a

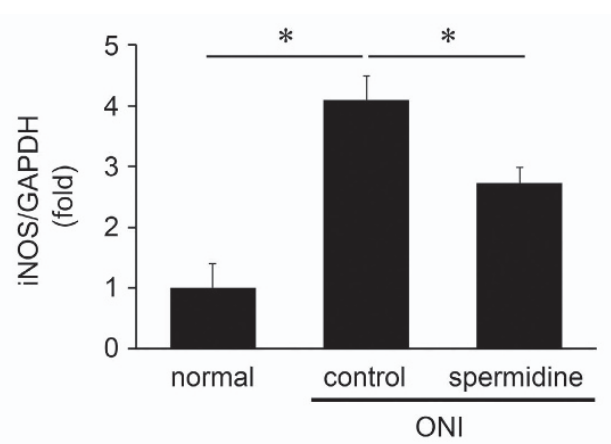

b
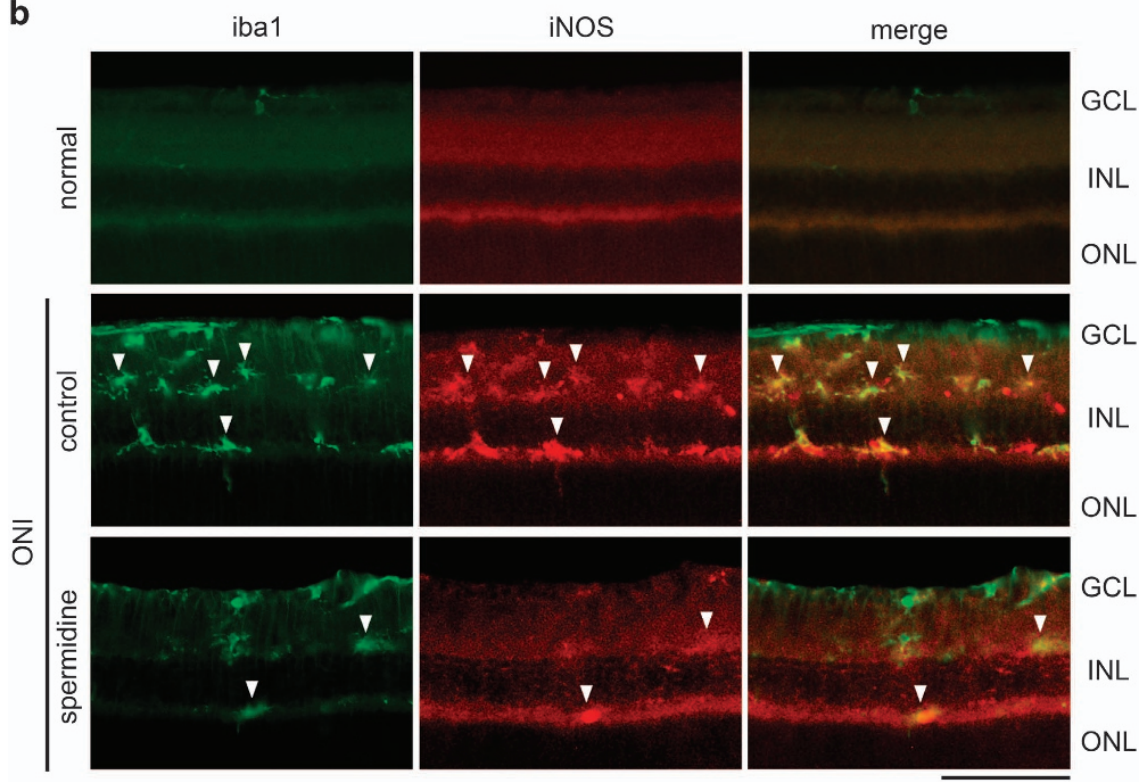

Figure 5 Effects of spermidine on ONI-induced iNOS activation in the retina. (a) mRNA expression levels of iNOS at d5 after ONI were determined using quantitative realtime PCR. Glyceraldehyde 3-phosphate dehydrogenase (GAPDH) was used as an internal control. The data are presented as means \pm S.E.M. of six samples for each experiment. ${ }^{*} P<0.05$. (b) Double-labeling immunohistochemistry of iba1 (green) and iNOS (red) at d5 after ONI. Overlapping immunoreactivities (yellow, arrowheads) indicate the activated microglial cells that produce iNOS. Scale bar: $100 \mu \mathrm{m}$

\section{Materials and Methods}

Mice. Experiments were performed using 8-week-old C57BL/6J mice (CLEA Japan, Tokyo, Japan) in accordance with the Tokyo Metropolitan Institute of Medical Science Guidelines for the care and use of animals.

Drug administration. Spermidine (Sigma, St Louis, MO, USA) was added to drinking water at $30 \mathrm{mM}$ for the treatment groups throughout the whole experimental period. ${ }^{21}$ The control group received normal drinking water. Drinking water was replaced every 2-3 days and spermidine was freshly added from $1 \mathrm{M}$ aqueous stock (spermidine/ $\mathrm{HCl}, \mathrm{pH} 7.4$ ), which was kept at $-20^{\circ} \mathrm{C}$ for no longer than 1 month.

ONI and anterograde labeling. Mice were anesthetized with sodium pentobarbital before ONI. Optic nerves were exposed intraorbitally and crushed at about $0.5-1.0 \mathrm{~mm}$ from the posterior pole of the eyeball, with fine surgical forceps for $5 \mathrm{~s}^{6,22}$ On d11 after ONI, $2 \mu \mathrm{g}$ of Alexa 488-conjugated CTB (Invitrogen, Carlsbad, CA, USA) was injected into the vitreous body. On d14 after ONI, the animals were perfused with Zamboni's Fixative ( $2 \%$ paraformaldehyde and $15 \%$ picric acid in $0.1 \mathrm{M}$ phosphate buffer). The optic nerve was removed, postfixed and immersed in $30 \%$ sucrose overnight at $4{ }^{\circ} \mathrm{C}$. The optic nerve was then embedded in an OCT compound (Sakura, Tokyo, Japan), frozen on dry ice and $10-\mu \mathrm{m}$ serial cross-sections were prepared using a cryostat and collected on MAS-coated glass slides (Matsunami, Osaka, Japan). To estimate the total number of regenerating axons, axonal outgrowth was quantified by counting CTB-positive axons that crossed a virtual line parallel to the lesion site at 250 and $500 \mu \mathrm{m}$ distal to the lesion site. ${ }^{23}$
Histological and morphometric studies. Paraffin-embedded retinal sections of $7 \mu \mathrm{m}$ thickness were cut through the optic nerve and stained with hematoxylin and eosin. The RGC number and the extent of retinal degeneration were quantified in two ways. ${ }^{54}$ First, the number of neurons in the GCL was counted from one ora serrata through the optic nerve to the other ora serrate. Second, in the same sections the thickness of the IRL (between the internal limiting membrane and the interface of the outer plexiform layer and the outer nuclear layer) was analyzed.

Imaging acquisition of spectral-domain OCT. Spectral-domain OCT (SD-OCT; RS-3000, Nidek, Aichi, Japan) examinations were performed at d0, 7 and 14 after ONI, with modifications. ${ }^{15,22}$ For fundus imaging, polymethyl methacrylate contact lenses optimal for mice (UNICON, Osaka, Japan) were placed on the corneas. Use of the contact lenses prevents anesthesia-induced cataract progression. A 60-D adaptor lens was placed on the objective lens of the Multiline OCT to focus on the mouse retina. For imaging of the IRLs on SD-OCT using the speckle noise-reduction method, line scans and a circular scan around the optic disc were performed. All the line scan images were location matched, scanning vertically through the center of the optic nerve head at three disc diameter lengths above the optic nerve head. All the circular scan images were obtained by scanning a circle centering around the optic nerve disc. The average thickness of GCC (between the internal limiting membrane and the interface of the inner plexiform layer and the inner nuclear layer) was measured. In this study, the maximum number of B-scans set by the manufacturer (50 for line scans and 10 for circular scans) was used for averaging. 

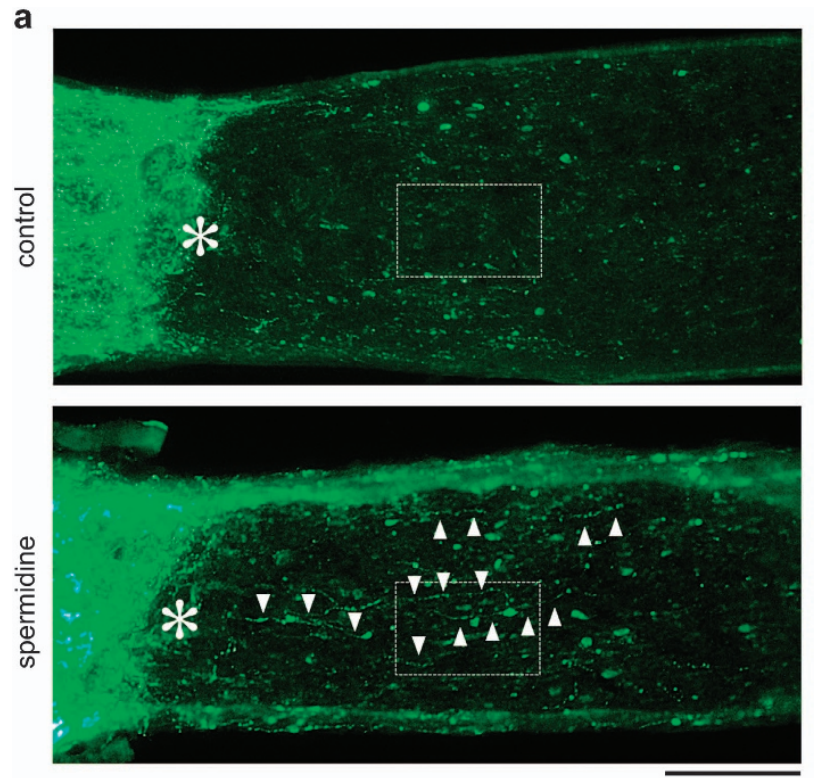

b

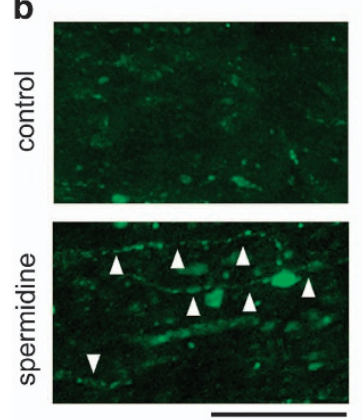

C

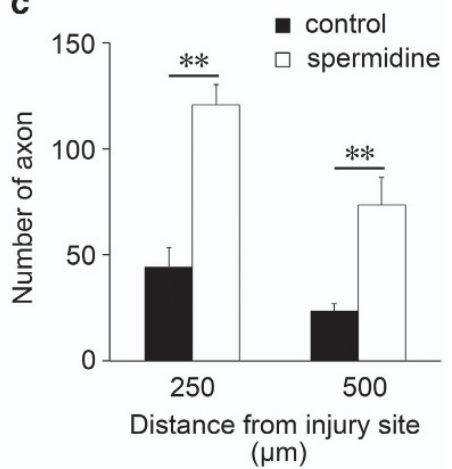

Figure 6 Effects of spermidine on optic nerve regeneration following ONI in vivo. (a) Longitudinal sections through the optic nerve showing CTB-positive axons distal to the injury site (*) in control and spermidine-treated mice at d14 after ONI. Arrowheads indicate regenerating axons. Scale bar: $100 \mu \mathrm{m}$. (b) Higher magnification of the images shown in a. Scale bar, $50 \mu \mathrm{m}$. (c) Quantitative analyses of regenerating axons extending 250 and $500 \mu \mathrm{m}$ from the injury site at d14 after ONI. The data are presented as means \pm S.E.M. of six samples for each experiment. ${ }^{* \star} P<0.01$

Immunohistochemistry. Retinas and optic nerves were examined by immunostaining as reported previously. ${ }^{6,47,55}$ Immunohistochemistry was performed using the following primary antibodies: phospho-p38 (1:500; V1211, Promega, Madison, WI, USA), NeuN (1: 1000, MAB377, Millipore, Billerica, MA, USA), iba1 $(1: 700 ; \text { ab5076, Abcam, Cambridge, MA, USA })^{22}$ and iNOS $(1: 100 ; 610328$, BD Biosciences, San Jose, CA, USA). Quantitative analysis of the IP cell number or stained region was carried out using BZ-H1C (Keyence Software, Osaka, Japan).

Immunoblot analysis. Immunoblotting was performed as reported previously. ${ }^{22}$ Membranes were incubated with an antibody against ASK1 (1:200; Cell Signaling Technology, Beverly, MA, USA), phospho-ASK1 (1:200; Cell Signaling), p38 (1:1000; BD Biosciences) or phospho-p38 (1:1000; BD Biosciences).

Quantitative real-time PCR. Quantitative RT-PCR was performed using the ABI 7500 fast RT-PCR system (Applied Biosystems, Foster City, CA, USA) with SYBR Green PCR Master Mix (Applied Biosystems) as reported previously. ${ }^{56}$ Complementary DNA reverse transcribed from total RNA was amplified by using primers specific for MCP-1 (sense: $5^{\prime}$-AACTGCATCTGCCCTAAGGT-3', antisense: 5'-ACGGGTCAACTTCACATTCA- $3^{\prime}$ ), MIP- $1 \alpha$ (sense: $5^{\prime}$-AGATTCCACGCCAATT CATC-3', antisense: 5'-CAGATCTGCCGGTTTCTCTT-3'), RANTES (sense:
5'-GCCCACGTCA AGGAGTATTT-3', antisense: 5'-TGACAAACACGACTGCAAGA $-3^{\prime}$ ), iNOS (sense: 5'-ACTGTGTGCCTGGAGGTTCT-3', antisense: 5'-GGCAGC CTCTTGTCTTTGAC-3') and glyceraldehyde 3-phosphate dehydrogenase (sense: 5'-TGCACCACCAACTGCTTAG-3', antisense: 5'-GGATGCAGGGATGATGTTC-3').

Statistics. For statistical comparison of two samples, we used a two-tailed Student's $t$-test. Data are presented as means \pm S.E.M. $P<0.05$ was regarded as statistically significant.

\section{Conflict of Interest}

The authors declare no conflict of interest.

Acknowledgements. We thank Mayumi Kunitomo and Keiko Okabe for their technical assistance. This study was supported by the Ministry of Education, Culture, Sports, Science and Technology of Japan (KN, AK, XG, CH and TH) and the Funding Program for Next Generation World-Leading Researchers (NEXT Program; TH).

1. Steinsapir KD, Goldberg RA. Traumatic optic neuropathy. Surv Ophthalmol 1994; 38: 487-518. 2. Sarkies N. Traumatic optic neuropathy. Eye (Lond) 2004; 18: 1122-1125.

3. Levin LA, Beck RW, Joseph MP, Seiff S, Kraker R. The treatment of traumatic optic neuropathy: the International Optic Nerve Trauma Study. Ophthalmology 1999; 106: 1268-1277.

4. Parrilla-Reverter G, Agudo M, Sobrado-Calvo P, Salinas-Navarro M, Villegas-Perez MP, Vidal-Sanz M. Effects of different neurotrophic factors on the survival of retinal ganglion cells after a complete intraorbital nerve crush injury: a quantitative in vivo study. Exp Eye Res 2009; 89: 32-41.

5. Park K, Luo JM, Hisheh S, Harvey AR, Cui Q. Cellular mechanisms associated with spontaneous and ciliary neurotrophic factor-cAMP-induced survival and axonal regeneration of adult retinal ganglion cells. J Neurosci 2004; 24: 10806-10815.

6. Namekata K, Harada C, Taya C, Guo X, Kimura H, Parada LF et al. Dock3 induces axonal outgrowth by stimulating membrane recruitment of the WAVE complex. Proc Natl Acad Sci USA 2010; 107: 7586-7591.

7. Tonari M, Kurimoto T, Horie T, Sugiyama T, Ikeda T, Oku H. Blocking endothelin-B receptors rescues retinal ganglion cells from optic nerve injury through suppression of neuroinflammation. Invest Ophthalmol Vis Sci 2012; 53: 3490-3500.

8. Quigley HA, Broman AT. The number of people with glaucoma worldwide in 2010 and 2020. Br J Ophthalmol 2006; 90: 262-267.

9. John SW, Smith RS, Savinova OV, Hawes NL, Chang B, Turnbull D et al. Essential iris atrophy, pigment dispersion, and glaucoma in DBA/2 J mice. Invest Ophthalmol Vis Sci 1998; 39: 951-962.

10. WoldeMussie E, Ruiz G, Wijono M, Wheeler LA. Neuroprotection of retinal ganglion cells by brimonidine in rats with laser-induced chronic ocular hypertension. Invest Ophthalmol Vis Sci 2001; 42: 2849-2855.

11. Garcia-Valenzuela E, Shareef S, Walsh J, Sharma SC. Programmed cell death of retinal ganglion cells during experimental glaucoma. Exp Eye Res 1995; 61: 33-44.

12. Morrison JC, Moore CG, Deppmeier LM, Gold BG, Meshul CK, Johnson EC. A rat model of chronic pressure-induced optic nerve damage. Exp Eye Res 1997; 64: 85-96.

13. Harada T, Harada C, Nakamura K, Quah HM, Okumura A, Namekata K et al. The potential role of glutamate transporters in the pathogenesis of normal tension glaucoma. J Clin Invest 2007; 117: 1763-1770.

14. Harada C, Namekata K, Guo X, Yoshida H, Mitamura Y, Matsumoto $Y$ et al. ASK1 deficiency attenuates neural cell death in GLAST-deficient mice, a model of normal tension glaucoma. Cell Death Differ 2010; 17: 1751-1759.

15. Semba K, Namekata K, Guo X, Harada C, Harada T, Mitamura Y. Renin-angiotensin system regulates neurodegeneration in a mouse model of normal tension glaucoma. Cell Death Dis 2014; 5: e1333.

16. Semba K, Namekata K, Kimura A, Harada C, Mitamura Y, Harada T. Brimonidine prevents neurodegeneration in a mouse model of normal tension glaucoma. Cell Death Dis 2014; 5 : e1341.

17. Laube G, Veh RW. Astrocytes, not neurons, show most prominent staining for spermidine/ spermine-like immunoreactivity in adult rat brain. Glia 1997; 19: 171-179.

18. Biedermann B, Skatchkov SN, Bringmann A, Pannicke T, Veh R, Bernstein H-G et al. Spermine/Spermidine is expressed by retinal glial (Müller) cells, and controls distinct $\mathrm{K}^{+}$ channels of their membrane. Glia 1998; 23: 209-220.

19. Rider JE, Hacker A, Mackintosh CA, Pegg AE, Woster PM, Casero RA Jr. Spermine and spermidine mediate protection against oxidative damage caused by hydrogen peroxide. Amino Acids 2007; 33: 231-240.

20. Eisenberg T, Knauer H, Schauer A, Buttner S, Ruckenstuhl C, Carmona-Gutierrez D et al. Induction of autophagy by spermidine promotes longevity. Nat Cell Biol 2009; 11: $1305-1314$.

21. Guo X, Harada C, Namekata K, Kimura A, Mitamura Y, Yoshida $H$ et al. Spermidine alleviates severity of murine experimental autoimmune encephalomyelitis. Invest Ophthalmol Vis Sci 2011; 52: 2696-2703. 
22. Katome T, Namekata K, Guo X, Semba K, Kittaka D, Kawamura K et al. Inhibition of ASK1p38 pathway prevents neural cell death following optic nerve injury. Cell Death Differ 2013; 20: $270-280$.

23. Semba K, Namekata K, Kimura A, Harada C, Katome T, Yoshida $\mathrm{H}$ et al. Dock3 overexpression and p38 MAPK inhibition synergistically stimulate neuroprotection and axon regeneration after optic nerve injury. Neurosci Lett 2014; 581: 89-93.

24. Kimura A, Namekata K, Guo X, Noro T, Harada C, Harada T. Valproic acid prevents NMDAinduced retinal ganglion cell death via stimulation of neuronal TrkB receptor signaling. Am J Pathol 2015; 185: 756-764.

25. Hattori K, Naguro I, Runchel C, Ichijo H. The roles of ASK family proteins in stress responses and diseases. Cell Commun Signal 2009; 7: 9.

26. Block ML, Zecca L, Hong JS. Microglia-mediated neurotoxicity: uncovering the molecular mechanisms. Nat Rev Neurosci 2007; 8: 57-69.

27. Brown GC, Neher JJ. Inflammatory neurodegeneration and mechanisms of microglial killing of neurons. Mol Neurobiol 2010; 41: 242-247.

28. Soga M, Matsuzawa A, Ichijo H. Oxidative stress-induced diseases via the ASK1 signaling pathway. Int J Cell Biol 2012; 2012: 439587.

29. Kawarazaki $\mathrm{Y}$, Ichijo $\mathrm{H}$, Naguro I. Apoptosis signal-regulating kinase 1 as a therapeutic target. Expert Opin Ther Targets 2014; 18: 651-664.

30. Kikuchi M, Tenneti L, Lipton SA. Role of p38 mitogen-activated protein kinase in axotomyinduced apoptosis of rat retinal ganglion cells. J Neurosci 2000; 20: 5037-5044.

31. Guo X, Harada C, Namekata K, Matsuzawa A, Camps M, Ji H et al. Regulation of the severity of neuroinflammation and demyelination by TLR-ASK1-p38 pathway. EMBO Mol Med 2010; 2: 504-515

32. Goyal A, Srivastava A, Sihota R, Kaur J. Evaluation of oxidative stress markers in aqueous humor of primary open angle glaucoma and primary angle closure glaucoma patients. Curr Eye Res 2014; 39: 823-829.

33. Inman DM, Lambert WS, Calkins DJ, Horner PJ. alpha-Lipoic acid antioxidant treatment limits glaucoma-related retinal ganglion cell death and dysfunction. PLOS One 2013; 8: e65389.

34. Rieck J. The pathogenesis of glaucoma in the interplay with the immune system. Invest Ophthalmol Vis Sci 2013; 54: 2393-2409.

35. Mosher Kl, Wyss-Coray T. Microglial dysfunction in brain aging and Alzheimer's disease. Biochem Pharmacol 2014; 88: 594-604.

36. Kaul M, Garden GA, Lipton SA. Pathways to neuronal injury and apoptosis in HIV-associated dementia. Nature 2001; 410: 988-994.

37. Yang G, Meng Y, Li W, Yong Y, Fan Z, Ding H et al. Neuronal MCP-1 mediates microglia recruitment and neurodegeneration induced by the mild impairment of oxidative metabolism. Brain Pathol 2011; 21: 279-297.

38. Skatchkov SN, Woodbury-Farina MA, Eaton M. The role of glia in stress: polyamines and brain disorders. Psychiatr Clin North Am 2014; 37: 653-678.

39. Williams K. Modulation and block of ion channels: a new biology of polyamines. Cell Signal 1997; 9: 1-13.

40. Inyushin MY, Huertas A, Kucheryavykh YV, Kucheryavykh LY, Tsydzik V, Sanabria P et al. L-DOPA uptake in astrocytic endfeet enwrapping blood vessels in rat brain. Parkinsons Dis-US 2012; 2012: 321406

41. Sala-Rabanal M, Li DC, Inyushin M, Skatchkov SN, Nichols CG. Polyamine transport by the polyspecific organic cation transporters OCT1, OCT2 and OCT3. Mol. Pharm. 2013; 10 1450-1458.

42. Hiasa M, Miyaji T, Haruna Y, Takeuchi T, Harada Y, Moriyama S et al. Identification of a mammalian vesicular polyamine transporter. Sci Rep 2014; 4: 6836.
43. Benedikt J, Inyushin M, Kucheryavykh YV, Rivera Y, Kucheryavykh LY, Nichols CG et al. Intracellular polyamines enhance astrocytic coupling. NeuroReport 2012; 23: 1021-1025.

44. Danesh-Meyer HV, Kerr NM, Zhang J, Eady EK, O'Carroll SJ, Nicholson LF et al. Connexin43 mimetic peptide reduces vascular leak and retinal ganglion cell death following retinal ischaemia. Brain 2012; 135: 506-520

45. Harada T, Harada C, Nakayama N, Okuyama S, Yoshida K, Kohsaka S et al. Modification of glial-neuronal cell interactions prevents photoreceptor apoptosis during light-induced retinal degeneration. Neuron 2000; 26: 533-541.

46. Harada T, Harada C, Kohsaka S, Wada E, Yoshida K, Ohno S et al. Microglia-Muller glia cell interactions control neurotrophic factor production during light-induced retinal degeneration. J Neurosci 2002; 22: 9228-9236.

47. Harada C, Guo X, Namekata K, Kimura A, Nakamura K, Tanaka K et al. Glia- and neuronspecific functions of TrkB signalling during retinal degeneration and regeneration. Nat Commun 2011; 2: 189

48. de Lima S, Koriyama Y, Kurimoto T, Oliveira JT, Yin Y, Li Y et al. Full-length axon regeneration in the adult mouse optic nerve and partial recovery of simple visual behaviors. Proc Natl Acad Sci USA 2012; 109: 9149-9154.

49. Namekata K, Kimura A, Kawamura K, Harada C, Harada T. Dock GEFs and their therapeutic potential: Neuroprotection and axon regeneration. Prog Retin Eye Res 2014; 43: 1-16.

50. Pernet V, Schwab ME. Lost in the jungle: new hurdles for optic nerve axon regeneration. Trends Neurosci 2014; 37: 381-387.

51. Deng K, He H, Qiu J, Lorber B, Bryson JB, Filbin MT. Increased synthesis of spermidine as a result of upregulation of arginase I promotes axonal regeneration in culture and in vivo. J Neurosci 2009; 29: 9545-9552.

52. Soda K, Kano Y, Sakuragi M, Takao K, Lefor A, Konishi F. Long-term oral polyamine intake increases blood polyamine concentrations. J Nutr Sci Vitaminol (Tokyo) 2009; 55: 361-366.

53. Bell MR, Belarde JA, Johnson HF, Aizenman CD. A neuroprotective role for polyamines in a Xenopus tadpole model of epilepsy. Nat Neurosci 2011; 14: 505-512.

54. Harada C, Nakamura K, Namekata K, Okumura A, Mitamura $Y$, lizuka $Y$ et al. Role of apoptosis signal-regulating kinase 1 in stress-induced neural cell apoptosis in vivo. Am J Pathol 2006; 168: 261-269.

55. Namekata K, Harada C, Guo X, Kimura A, Kittaka D, Watanabe H et al. Dock3 stimulates axonal outgrowth via GSK-3beta-mediated microtubule assembly. J Neurosci 2012; 32: 264-274.

56. Guo X, Nakamura K, Kohyama K, Harada C, Behanna HA, Watterson DM et al. Inhibition of glial cell activation ameliorates the severity of experimental autoimmune encephalomyelitis. Neurosci Res 2007: 59: 457-466.

(i) Cell Death and Disease is an open-access journal published by Nature Publishing Group. This work is licensed under a Creative Commons Attribution 4.0 International License. The images or other third party material in this article are included in the article's Creative Commons license, unless indicated otherwise in the credit line; if the material is not included under the Creative Commons license, users will need to obtain permission from the license holder to reproduce the material. To view a copy of this license, visit http://creativecommons.org/licenses/by/4.0/ 